\title{
Tasa de sedimentos en suspensión por efectos de un vertedor lateral en un río de planicie
}

\section{Suspended sediment rate due to effects of a lateral spillway in a plain river}

\author{
Roberto Rodríguez-Bastarmérito ${ }^{1 *}$, Juan Arcadio Saiz-Hernández ${ }^{2}$, Dagoberto Burgos-Flores², Víctor Hugo \\ Guerra-Cobian $^{3}$, Liliana Lizarraga-Mendiola ${ }^{4}$, Ruperto Ortíz-Gómez ${ }^{5}$ \\ ${ }^{1}$ Universidad Juárez Autónoma de Tabasco, División Académica de Ingeniería y Arquitectura. Carretera Cunduacán-Jalpa de \\ Méndez, Km 1, Col. La Esmeralda, CP. 86690. Cunduacán, Tabasco, México. \\ ${ }^{2}$ Universidad de Sonora. Blvd. Luis Encinas y Rosales s/n, Col. Centro, Hermosillo, Sonora, México. \\ ${ }^{3}$ Universidad Autónoma de Nuevo León. Pedro de Alba s/n, Ciudad Universitaria, San Nicolás de Los Garza, Nuevo León, \\ México. \\ ${ }^{4}$ Universidad Autónoma del Estado de Hidalgo. Carretera Pachuca Tulancingo, CP. 42184, Pachuca de Soto, Hidalgo, México. \\ ${ }^{5}$ Universidad de Zacatecas, Jardín Juárez 147, CP. 98000. Centro, Zacatecas, México. \\ ${ }^{*}$ Autor de correspondencia: robertrb71@hotmail.com
}

Artículo científico recibido: 02 de abril de 2017 aceptado: 11 de octubre de 2017

RESUMEN. Los canales de desvío y vertedores laterales en un río de planicie, derivan los flujos líquidos excedentes, reduciendo los niveles y evitando el desbordamiento de los cauces, lo que mitiga las inundaciones. El estudio tuvo el objetivo de determinar la tasa de sedimentos en suspensión por efectos de un vertedor lateral en un río de planicie. El funcionamiento de estas estructuras cambia el comportamiento del flujo líquido y de los sedimentos que transporta en suspensión, provocando cambios en la morfología e hidrodinámica del cauce. Se utilizó un perfilador de flujo Doppler (ADCP) River Ray y un muestreador integral de sedimentos en suspensión US-DH59, para medir los flujos líquidos y sólidos en el río de la Sierra en el Estado de Tabasco, México y en el vertedor lateral El Censo. Los datos obtenidos en campo se utilizaron para evaluar los efectos del vertedor lateral sobre la hidrodinámica y morfología del cauce. Los resultados muestran que hay un incremento de $109.4 \%$ en la tasa de sedimentos en el flujo aguas abajo del vertedor y desequilibrio geomorfológico, lo que denotan una alteración al comportamiento natural del flujo bifásico compuesto por una fase de flujo líquido y otra de flujo de sedimentos.

Palabras clave: Flujo bifásico, inundaciones, hidrodinámica, morfología, transporte de sedimentos

ABSTRACT. Diversion canals and lateral spillways in a plain river divert surplus liquid flows, reducing the levels and avoiding the overflow of the channels, which mitigates flooding. The objective of the study was to determine the suspended sediment rate due to the effects of a lateral spillway in a plain river. The operation of these structures changes the behavior of the liquid flow and the sediments that it transports in suspension, causing changes in the morphology and hydrodynamics of the channel. A RiverRay Acoustic Doppler Current Profiler (ADCP) and a US-DH59 depth-integrating suspended sediment sampler were used to measure the liquid and solid flows in the Sierra River in the State of Tabasco, Mexico and in the El Censo lateral spillway. The data obtained in the field were used to evaluate the effects of the lateral spillway on the hydrodynamics and morphology of the channel. The results show a $109.4 \%$ increase in the sediment rate in the flow downstream of the spillway and geomorphological imbalance, which denote an alteration to the natural behavior of the biphasic flow composed of a liquid flow phase and a sediment flow one.

Key words: Biphasic flow, floods, hydrodynamics, morphology, sediment transport 


\section{INTRODUCCIÓN}

En el diseño de obras de protección contra inundaciones se ha dado prioridad a los principios hidrológicos, ignorando el transporte de sedimentos (Chen y Sawin 2014), no obstante este proceso constituye un factor determinante en la estabilidad y el funcionamiento hidráulico de un río (Fan et al. 2008, Csiki y Rhoads 2014). Cuando se modifica el cauce de un río, en la mayoría de los casos se provoca un desequilibrio geomorfológico, como la construcción de infraestructuras lineales que se realizan para proteger zonas urbanas del desbordamiento de los ríos de planicies, durante las avenidas máximas (Naoura y Bennabitade 2011). Pero se ha observado que en el mediano plazo, estas estructuras generan un aumento en los niveles, provocando de nuevo inundaciones, que cambian las condiciones de descarga y causan alteraciones en la hidrodinámica y geomorfología del río (Fan et al. 2008, Fahmy 2015).

Los fenómenos que interactúan en los procesos fluviales en las estructuras de derivación, son complejos y se caracterizan por la presencia de flujos que varían de subcríticos a supercríticos (Arega et al. 2008). Debido a la complejidad de los fenómenos, se deben observar como un sistema, por lo que para su comprensión y análisis se elaboran modelos numéricos, que permitan reproducir los procesos que ocurren al construir una estructura lateral de derivación y las tendencias evolutivas de su cauce (Minea y Zaharia 2011). Además de tomar en cuenta las variables de flujo, de sedimentos, y los efectos por la variación del flujo líquido, que condicionan la capacidad de transporte de sedimentos (Rosier et al. 2011, Li et al. 2014). Como alternativa para comprender y representar estos procesos de hidráulica fluvial, se tienen modelos bidimensionales, calibrados y validados con datos obtenidos en campo (Domeneghetti et al. 2014), que generan modelos numéricos que simulan el comportamiento hidrodinámico de las estructuras de derivación o modelos físicos reducidos (CONAGUA 2008). Los cuales también ayudan al entendimiento del funcionamiento hidráulico, aunque la dificultad para representar el transporte de sedimentos puede complicar el diseño (Dutta y Sen 2016). Por lo anterior, el objetivo del trabajo fue determinar el transporte de sedimentos y la distribución de los flujos líquidos y sólidos entre el río de la Sierra y el vertedor de derivación El Censo.

\section{MATERIALES Y MÉTODOS}

El sitio de estudio se localizó en la región hidrológica RH30, cuenca rio Grijalva-Villahermosa $\mathrm{RH} 30 \mathrm{D}$, en el río de la Sierra en el estado de Tabasco. Pertenece a la subcuenca RH30Di, la cual tiene un área de $1073.57 \mathrm{Km}^{2}$. El río de la Sierra vierte sus aguas en el rio Grijalva, atravesando la zona urbana de la ciudad de Villahermosa. El estudio se realizó en un tramo del cauce de $2.5 \mathrm{~km}$, entre el sitio Pueblo Nuevo 1 (PN1) localizado a los $17.872291^{\circ} \mathrm{LN}$ y $-92.869180^{\circ} \mathrm{LO}$, y el sitio Pueblo Nuevo 2 (PN2) localizado a los $17.880599^{\circ}$ LN y $-92.873369^{\circ}$ LO.

Se utilizó un perfilador de flujo Doppler (ADCP) River Ray y un muestreador integral de sedimentos en suspensión US-DH59 para medir los flujos de líquidos y sólidos. Fotografías aéreas y cartografía hidrológica y de infraestructura del INEGI (2010a, 2010b), datos hidrométricos de la estación hidrométrica de Pueblo Nuevo, localizada a $17^{\circ} 51^{\prime}$ $55.00^{\prime \prime}$ LN y $92^{\circ} 52^{\prime} 8.00^{\prime \prime}$ LO, los niveles de la superficie libre del agua y los registros de aforo líquido diario del 2012 y 2013 de la CONAGUA (2016), información del proyecto del vertedor lateral de derivación del sitio de estudio y los informes técnicos del diseño e implementación de los modelos físicos de estas estructuras elaborados por el Instituto de Ingeniería de la UNAM (CONAGUA 2008). Con la información recopilada, se elaboró un sistema de información geográfica que se utilizó para realizar el análisis hidrodinámico y de transporte de sedimentos.

\section{Secciones de aforo}

Para caracterizar la zona, se realizaron recorridos por tierra y agua, identificando tres sitios con características hidráulicas adecuadas para medir 


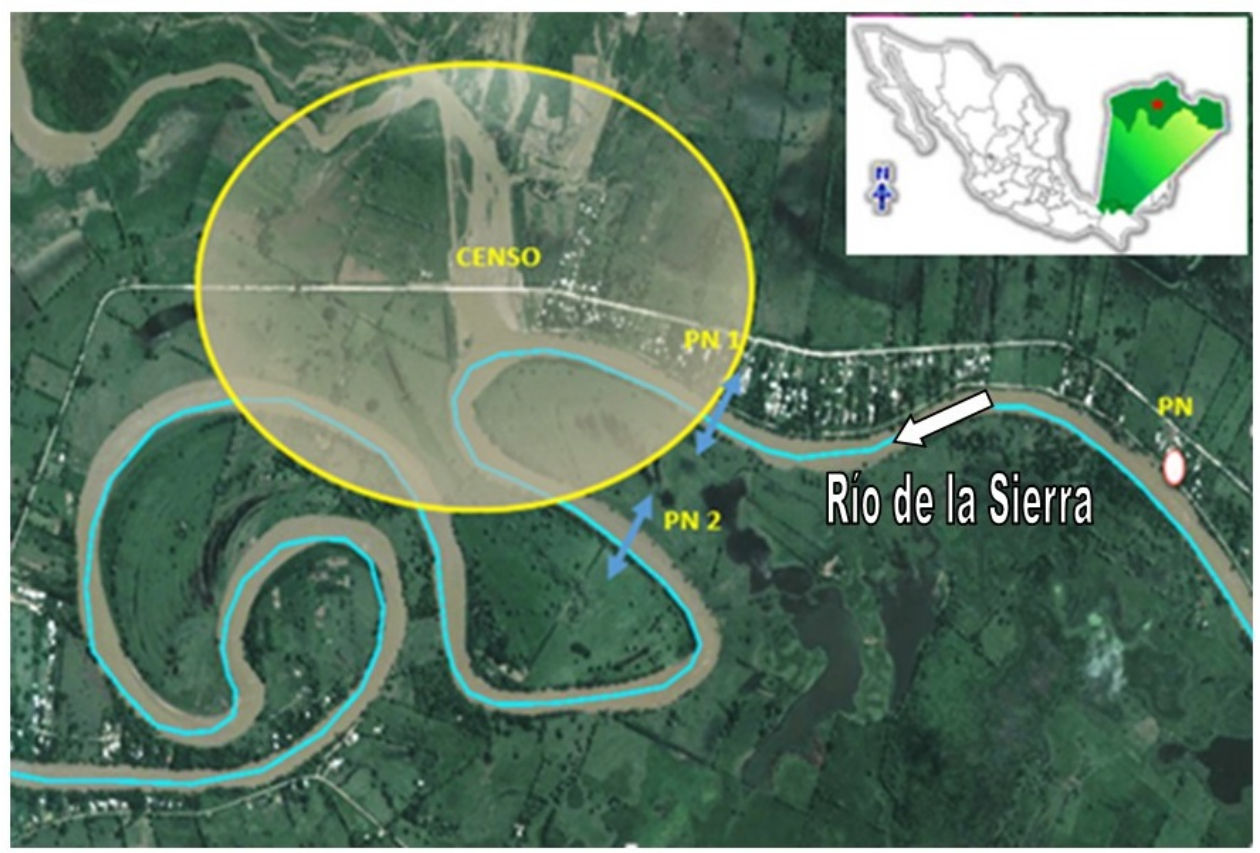

Figura 1. Localización del sitio de estudio.

los gastos de líquido y sólido. La primera se localiza en la estación de aforo PN1, la segunda en un tramo recto del río a una distancia de un kilómetro del vertedor lateral de derivación en el sitio denominado PN2 y la tercera sobre el vertedor lateral Censo (Figura 1).

\section{Aforo de gasto líquido}

El gasto líquido se midió con el perfilador de corriente acústico Doppler RiverRay (ADCP), que se encuentra acoplado a un trimarán de $1.2 \mathrm{~m}$ de largo, $0.80 \mathrm{~m}$ de ancho y $0.20 \mathrm{~m}$ de alto. El ADCP funciona con intercomunicación inalámbrica con frecuencia de radio o vía bluetooth, con rango de operación de 0.40 hasta $60 \mathrm{~m}$ de profundidad. Con el equipo se obtiene el espectro de velocidades y la batimetría de la sección transversal. El gasto líquido, la velocidad y la profundidad, se obtuvieron con el promedio de seis medidas con variación menor al $5 \%$. Los resultados se validaron comparándolos con los datos medidos por la Comisión Nacional de Agua en la estación Pueblo Nuevo.

\section{Muestreo de sólidos suspendidos}

La variación de la concentración de sedimentos en la sección transversal, se determinó con el muestreador integral US-DH-59, aplicando el método ETR, que determina el transporte de sedimentos mediante un volumen de muestra proporcional a la cantidad de flujo para cada vertical (Hudson 1997). El muestreador integrado en cada vertical a la profundidad del río (US-DH-59) posee una válvula que controla el acceso del agua, para obtener muestras representativas de una vertical de medición. Para lo cual se hizó descender el muestreador hasta el fondo del río y se regresó a la superficie en una vertical de medición a velocidad constante, que estuvo en función de la velocidad de la corriente, la profundidad y el diámetro de la boquilla del muestreador, para tener una muestra integrada (Figura 2).

\section{Concentración de sedimentos}

La concentración de sedimentos se determinaron cuantificando la relación de unidades de masa 


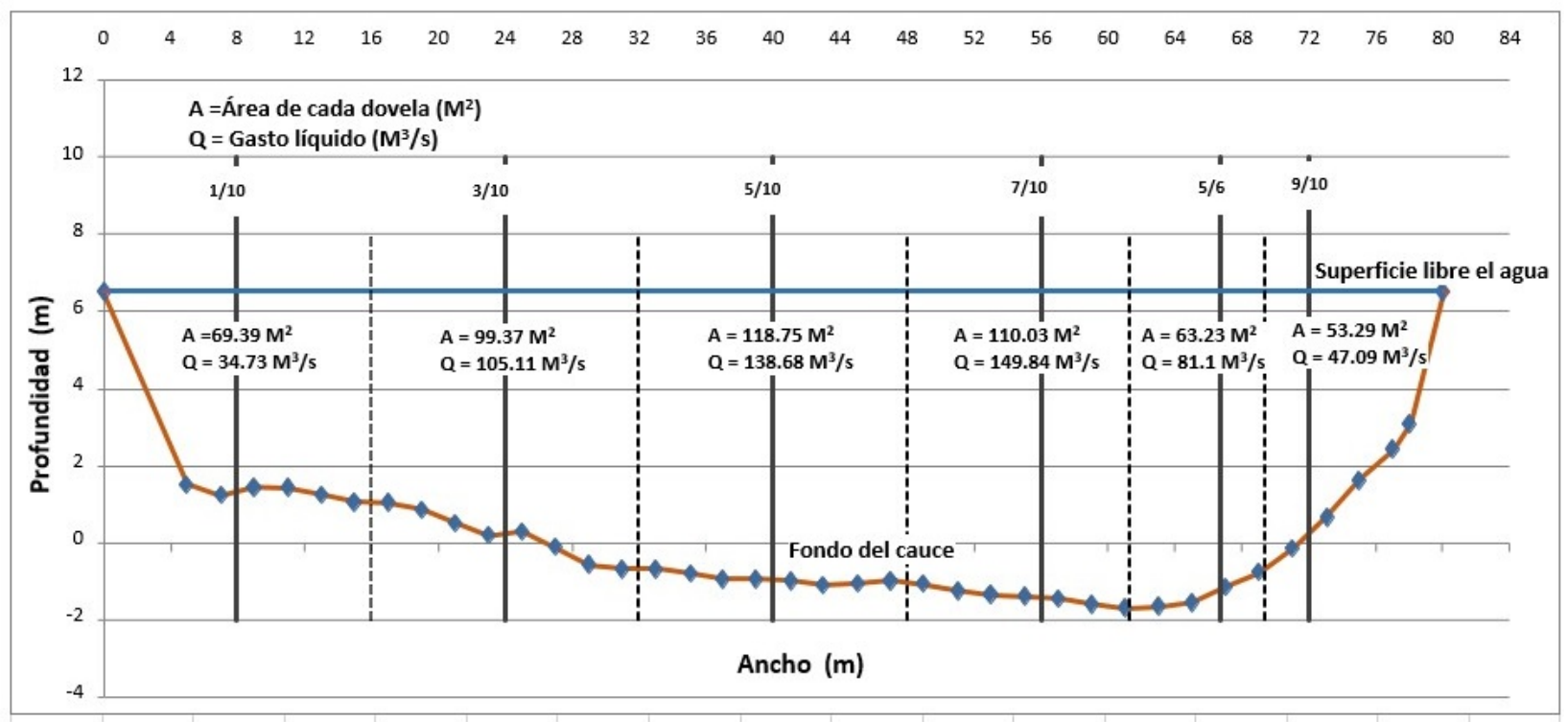

Figura 2. Sección del cauce para determinar el transporte de flujo sólido con el método ETR.

de sólidos entre el volumen $\left(\mathrm{kg} \mathrm{m}^{3}\right)$, con la ecuación $C_{s}=M_{m} / V_{m}$. Donde: $C_{s}$ representa concentración de sedimentos, $M_{m}$ la masa seca de la muestra y $\mathrm{V}_{m}$, el volumen de la muestra.

La determinación de la masa de sedimento se realizó de acuerdo con la norma NMX-AA-034SCFI-2015 (DOF 2015), para lo cual se uso una cápsula de porcelana a peso constante, en la que se colocó muestra homogenizada de sedimientos, para luego evaporar en un horno de secado a 105 ${ }^{\circ} \mathrm{C}$ hasta peso constante, y pesar la cápsula en una balanza analítica con precisión de $0.0001 \mathrm{~g}$.

\section{Flujos de sedimentos}

Para aplicar el método ETR y determinar el transporte de sedimentos en suspensión, se dividió el ancho del río, evaluando el transporte de sedimentos por dovela, para lo cual se realizó la medición para evaluar la concentración de sedimentos en distintas verticales; obteniendo una variedad de dovelas, de la cual se consideró aquella que permitía optimizar los tiempos de medición sin inconsistencias. Luego, se sumaron los gastos correspondientes de cada una de las dovelas para obtener el transporte total de sedimentos en suspensión. Para lo cual se obtuvieron las áreas hidráulicas y las velocidades de cada dovela, con el perfilador acústico doppler de corriente ADCP River Ray. Con lo que se obtuvo el gasto líquido de cada dovela al multiplicar el área por la velocidad, con la ecuación:

$$
Q_{l i}=V_{i} * A_{i(A D C P)}
$$

Una vez obtenido el gasto líquido correspondiente a cada dovela y su correspondiente concentración de sedimentos, se obtuvo el gasto sólido como el producto de ambos. El flujo total de sedimentos $\left(Q_{s t}\right)$, se obtuvo de la suma del flujo de sedimentos en cada dovela $Q_{s i}$, con la ecuación:

$$
Q_{s t}=\sum_{i=1}^{n} Q_{s i}(A D C P)
$$

Dónde: $\mathrm{Q}_{l i}$ flujo líquido por dovela, $\mathrm{A}_{i}$ área hidráulica por dovela, corresponden al área hidráulica obtenida con el equipo $A D C P, V_{i}$ velocidad promedio en la dovela utilizando el ADCP, $Q_{s i}$ flujo de sedimentos por dovela, $C_{s i}$ representa concentración de sedimentos, y $Q_{s t}$ flujo de sedimentos total. 
Tabla 1. Distribución de flujos líquidos y sólidos.

\begin{tabular}{|c|c|c|c|c|c|c|c|c|c|c|}
\hline \multirow[t]{3}{*}{ Medida } & \multicolumn{3}{|c|}{ PN1 } & \multicolumn{3}{|c|}{ Censo } & \multicolumn{3}{|c|}{ PN2 } & \multirow{3}{*}{$\begin{array}{c}\text { Escala PN1 } \\
\begin{array}{c}\text { m.s.n.m } \\
(\mathrm{m})\end{array}\end{array}$} \\
\hline & $\mathrm{QL}$ & QS & CS & $\mathrm{QL}$ & QS & CS & $\mathrm{QL}$ & QS & CS & \\
\hline & $\mathrm{m}^{3} \mathrm{~s}^{-1}$ & $\mathrm{Kg} \mathrm{s}^{-1}$ & $\mathrm{Kg} \mathrm{m}^{3}$ & $\mathrm{~m}^{3} \mathrm{~s}^{-1}$ & $\mathrm{Kg} \mathrm{s}^{-1}$ & $\mathrm{Kg} \mathrm{m^{3 }}$ & $\mathrm{m}^{3} \mathrm{~s}^{-1}$ & $\mathrm{Kg} \mathrm{s}^{-1}$ & $\mathrm{Kg} \mathrm{m^{3 }}$ & \\
\hline 1 & 109.20 & 17.84 & 0.16 & 0.00 & 0.00 & 0.00 & 109.20 & 17.84 & 0.16 & 3.24 \\
\hline 2 & 192.72 & 159.21 & 0.83 & 0.00 & 0.00 & 0.00 & 192.72 & 159.21 & 0.83 & 4.27 \\
\hline 3 & 153.41 & 31.38 & 0.20 & 0.00 & 0.00 & 0.00 & 153.41 & 31.38 & 0.20 & 3.96 \\
\hline 4 & 346.96 & 188.78 & 0.54 & 3.99 & 2.16 & 0.54 & 342.97 & 186.62 & 0.54 & 5.63 \\
\hline 5 & 346.69 & 312.24 & 0.90 & 2.40 & 1.63 & 0.68 & 344.29 & 310.61 & 0.90 & 5.62 \\
\hline 6 & 440.59 & 968.99 & 2.20 & 63.07 & 63.44 & 1.01 & 377.52 & 905.56 & 2.40 & 5.99 \\
\hline 7 & 348.84 & 345.78 & 0.99 & 9.00 & 6.36 & 0.71 & 339.84 & 339.42 & 1.00 & 5.68 \\
\hline 8 & 557.23 & 1432.76 & 2.57 & 149.55 & 161.19 & 1.08 & 407.68 & 1080.84 & 2.65 & 6.33 \\
\hline 9 & 441.49 & 350.91 & 0.79 & 87.19 & 55.89 & 0.64 & 354.30 & 295.01 & 0.83 & 6.08 \\
\hline 10 & 497.61 & 1693.33 & 3.40 & 140.73 & 220.28 & 1.57 & 356.88 & 1325.75 & 3.71 & 6.34 \\
\hline 11 & 568.80 & 516.97 & 0.91 & 218.30 & 148.49 & 0.68 & 350.50 & 331.63 & 0.95 & 6.52 \\
\hline 12 & 585.26 & 546.51 & 0.93 & 265.63 & 182.86 & 0.69 & 319.63 & 315.65 & 0.99 & 6.66 \\
\hline 13 & 516.08 & 482.41 & 0.93 & 228.26 & 157.21 & 0.69 & 287.82 & 292.68 & 1.02 & 6.52 \\
\hline 14 & 447.49 & 343.46 & 0.77 & 131.90 & 83.25 & 0.63 & 315.59 & 252.70 & 0.80 & 6.22 \\
\hline
\end{tabular}

\section{RESULTADOS}

Se obtuvieron los flujos líquidos y sólidos, así como la concentración de sedimentos en el río de la Sierra en el sitio PN1, sobre el río en el vertedor lateral, sobre la derivación lateral el Censo en la margen derecha y aguas abajo en el punto PN2. En la Tabla 1, se presentan los resultados obtenidos en las 14 mediciones de flujos líquidos $(\mathrm{QL})$, flujo sólido $(\mathrm{QS})$ y la concentración de sedimentos (CS). Cuando los niveles del río superan los $5.50 \mathrm{msnm}$, el vertedor lateral deriva flujo líquido y ocurre disminución del porcentaje de flujo líquido en la sección PN2 con respecto a la sección PN1, situación opuesta ocurre en el vertedor, ya que cuando aumenta el flujo líquido en PN1, se observa mayor porcentaje derivado en el vertedor el Censo (Figura 3). La tasa promedio de cambio de flujo líquido es de 0.4694 entre PN1 y PN2, y de 0.9851 entre PN1 y el Censo; situación opuesta se presenta en el comportamiento de los sólidos (Figura 4). En la sección PN2, se observa que el paso del flujo de sedimentos en su concentración se incrementa, con el aumento en el flujo líquido que se deriva por el vertedor lateral, con tasa promedio de cambio de flujo sólido de 0.7743 entre PN1 y PN2, y de 0.1065 entre PN1 y Censo.

En la Figura 5 se observa la relación lineal del flujo líquido y la concentración de sedimentos, se a- precia que el río, a su paso por la estación de aforo PN1, transporta sedimentos con una tasa promedio de cambio de 0.0032 en relación al gasto líquido $\mathrm{y}$, después de la derivación de flujos sobre el vertedor lateral, cambia a 0.0067 , con incremento del $109.4 \%$. En el flujo derivado por el vertedor lateral del Censo se observa un incremento en la tasa de cambio, lo que contrasta con la mayor proporción de caudal líquido que se deriva, por lo que influye en la mayor cantidad de sedimentos transportados.

\section{DISCUSIÓN}

Los resultados concinciden con los obtenidos en estudios de vertedores laterales en ríos de planicie, que fueron construidos con el propósito de mitigar inundaciones por desbordamiento, lo que confirma, que se reduce el nivel de la superficie libre del agua del río (CONAGUA 2008). Lo que contribuye para evitar inundaciones, debido a que los vertedores alteran la distribución de flujos líquidos y de sedimentos, generando mayor concentración de sedimentos sobre el río aguas abajo del vertedor lateral (Rosier et al. 2011, Li W et al. 2014, Arnaud et al. 2015). La concentración de sedimentos determina la forma del fondo del cauce, debido a la disminución de la capacidad de transporte de sedimentos, lo que causa un aumento en la deposición de sedimentos, particularmente aguas abajo del ver- 


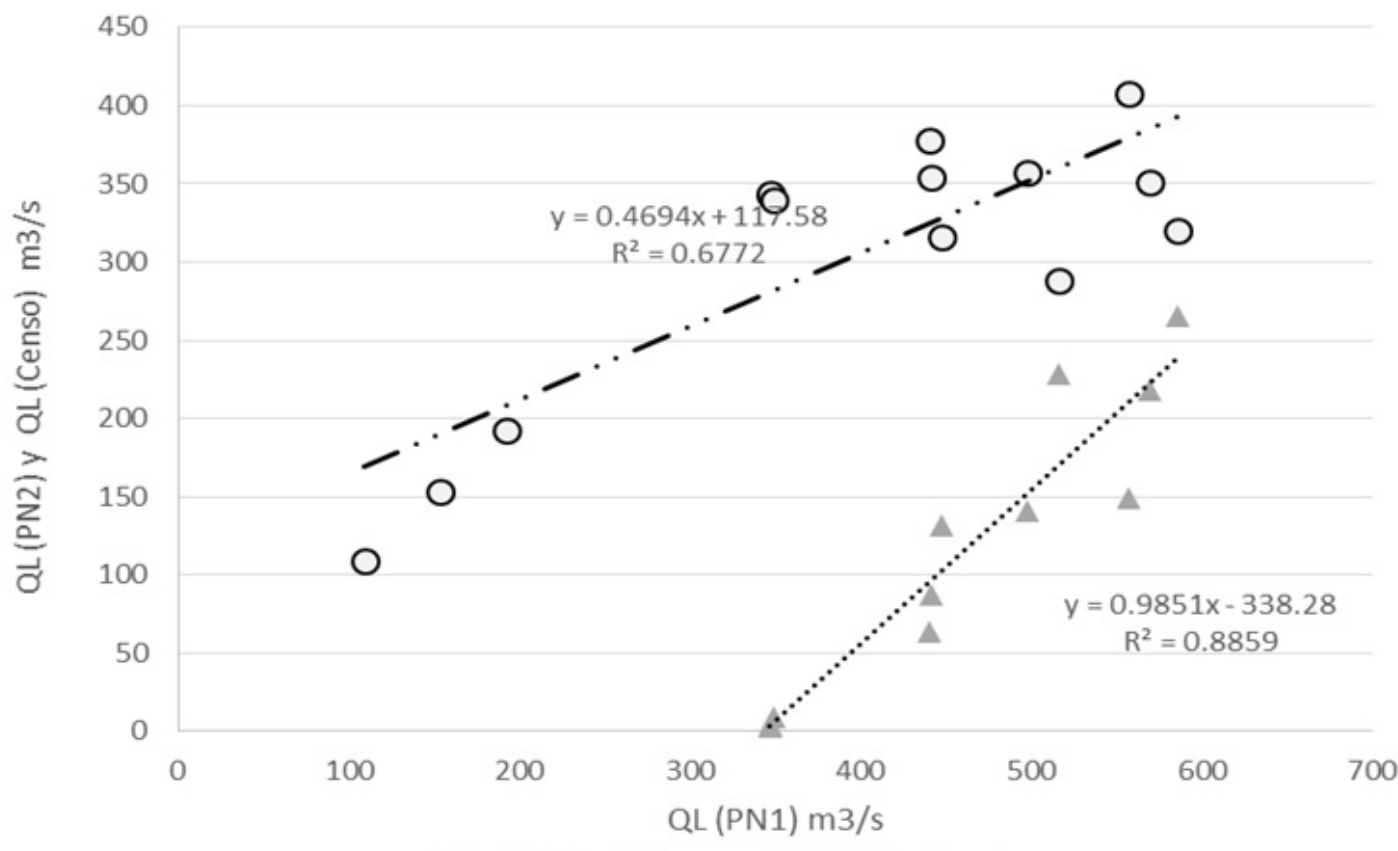

O QL (PN1 Vs PN2) $\quad$ QL (PN1 Vs Censo)

Figura 3. Relación de flujos líquidos de PN1 con las estaciones PN2 y Censo.

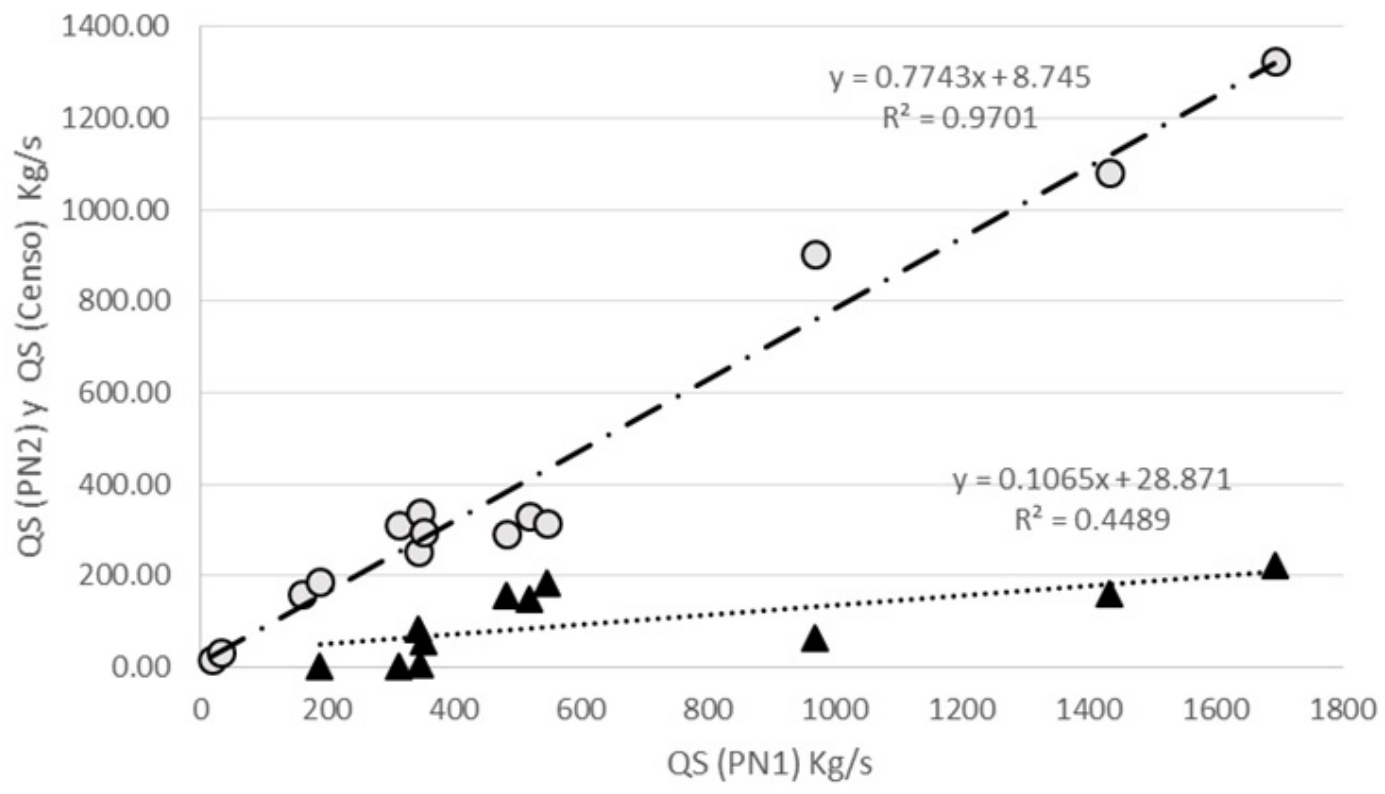

$\boldsymbol{\Delta}$ QS (PN1 Vs Censo) O QS(PN1 Vs PN2)

Figura 4. Relación de flujos sólidos de PN1 con las estaciones PN2 y Censo. 

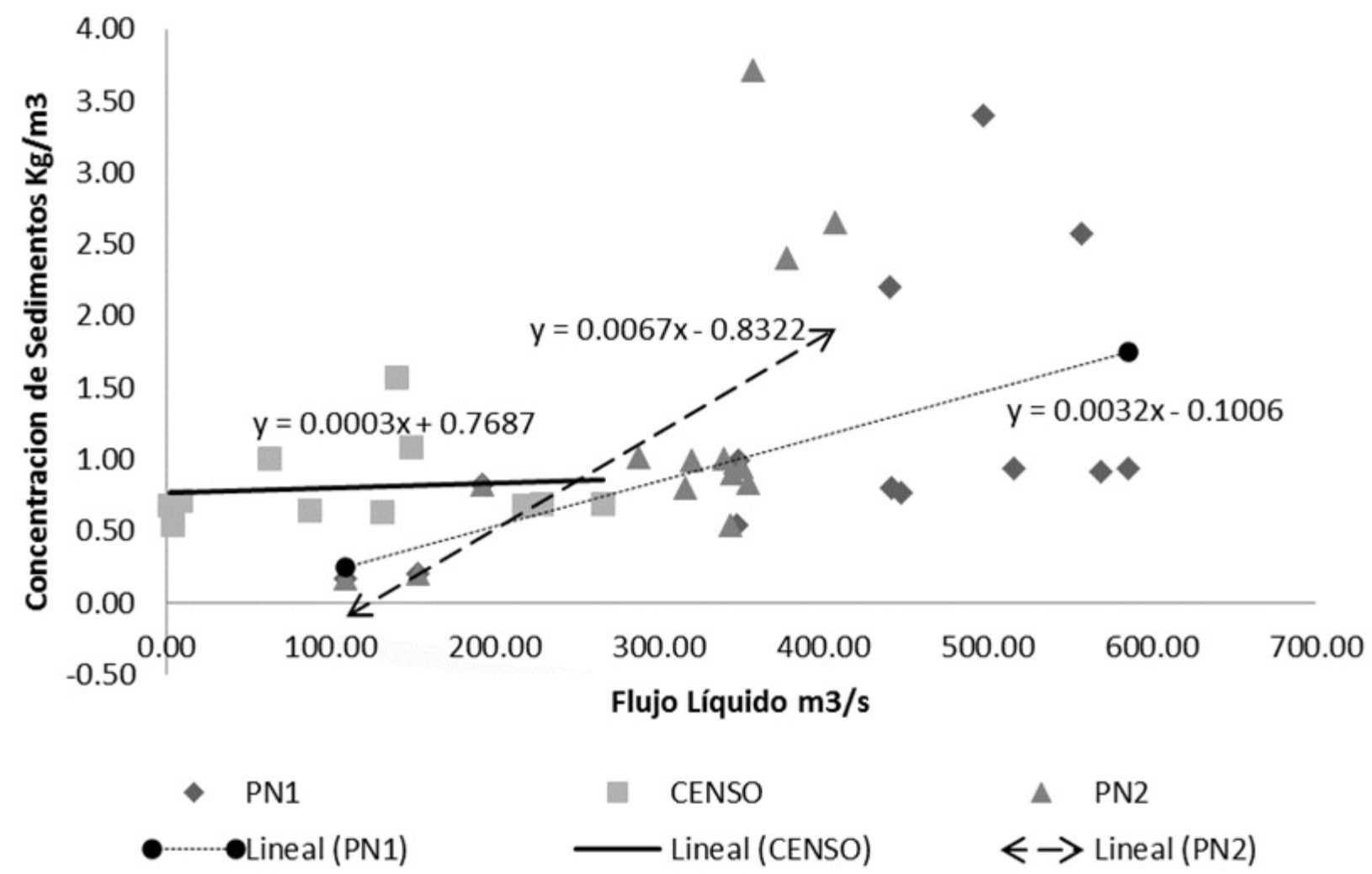

Figura 5. Distribución de flujos líquidos y sólidos.

tedor lateral. Esto mismo fue reportado por Bhuiyan et al. (2007) y Wohl (2015) quienes indican que los vertedores de derivación, modifican los patrones de sedimentos en los ríos y en el caso de los ríos de planicie, cuando las derivaciones de los flujos excedentes se conducen hacia las llanuras de inundación, provocan cambios en la hidrodinámica del río (Rosier et al. 2011). Los cambios en la distribución de los sedimentos observados, provocan aumento en la concentración de sedimentos que continúan por el río después de la derivación lateral, lo que afecta el funcionamiento hidráulico del río, al respecto Fan et al. (2008) indican que esto produce aumento del nivel del río, y provoca inundaciones.

\section{CONCLUSIONES}

Se obtuvo la tasa de cambio de la tasa de sedimentos en suspensión, por efectos del vertedor lateral en un río, lo que provoca cambios en la morfología e hidrodinámica del cauce. La modificación de los patrones de hidrodinámica y el transporte de sedimentos, generan importantes volúmenes de deposición de sedimentos que alteran el funcionamiento natural del río, lo que se hace más notable entre la escotadura y la continuidad del río en la sección de aforo PN2, donde se tiene mayor concentración de sedimentos. Se tuvo interdependencia entre comportamiento de los flujos líquidos y de sedimentos ligado a las condiciones fisiográficas del cauce, la cuenca y el volumen líquido transportado, que da lugar al flujo bifásico con las características específicas de un río de planicie. 


\section{LITERATURA CITADA}

Arega F, Lee JH, Tang HW (2008) Hydraulic jet control for river junction design of Yuen Long Bypass Floodway, Hong Kong. Journal of Hydraulic Engineering 134: 23-33.

Arnaud F, Piégay H, Schmitt L, Rollet AJ, Ferrier V, Béal D (2015) Historical geomorphic analysis (19322011) of a by-passed river reach in process-based restoration perspectives: The Old Rhine downstream of the Kembs diversion dam (France, Germany). Geomorphology 236: 163-177.

Bhuiyan F, Hey RD, Wormleaton PR (2007) Hydraulic evaluation of W-Weir for River restoration. Journal of Hydraulic Engineering 133: 596-609.

Chen H, Swain A (2014) The grand Ethiopian renaissance Dam: evaluating its sustainability standard and geopolitical significance. Energy Development Frontier 3: 11-19

CONAGUA (2008) Plan hídrico integral de Tabasco (PHIT) - Primera Etapa 2008, Estudio solicitado por el Ejecutivo Federal a través de la Comisión Nacional del Agua a la UNAM por conducto del Instituto de Ingeniería. http://www.gob.mx/conagua/documentos/plan-hidrico-integral-de-tabasco-phit. Fecha de consulta 19 de febrero del 2018.

CONAGUA (2016) Banco Nacional de Datos de Aguas Superficiales, Gerencia de Aguas Superficiales e Ingeniería de Ríos (GASIR), de la Comisión Nacional del Agua. http://www.conagua.gob.mx/CONAGUA07/ Contenido/Documentos/Portada\%20BANDAS.htm. Fecha de consulta 19 de febrero del 2017.

Csiki SJ, Rhoads BL (2014) Influence of four run-of-river dams on channel morphology and sediment characteristics in Illinois, USA. Geomorphology 206: 215-229.

DOF (2015) Norma Mexicana NMX-AA-034-SCFI-2015. Análisis de agua - medición de sólidos y sales disueltas en aguas naturales, residuales y residuales tratadas - método de prueba (cancela a NMX-AA034-SCFI-2001)

Domeneghetti A, Tarpanelli A, Brocca L, Barbetta S, Moramarco T, Castellarin A, et al. (2014) The use of remote sensing-derived water surface data for hydraulic model calibration. Remote Sensing of Environment 149: 130-141.

Dutta S, Sen D (2016) Sediment distribution and its impacts on Hirakud Reservoir (India) storage capacity. Lakes \& Reservoirs: Research \& Management 21: 245-263.

Fan P, Li JC, Liu QQ, Singh VP (2008) Case study: influence of morphological changes on flooding in Jingjiang River. Journal of Hydraulic Engineering 13: 1757-1766.

Fahmy MR (2015) Effect of sediment deposition on the efficiency of Fayoum weir. Flow Measurement and Instrumentation 46: 133-138.

Hudson NW (1997) Medición sobre el terreno de la erosión del suelo y de la escorrentía. Organización de las Naciones Unidas para la Agricultura y la Alimentación. Roma, Italia. http://www.fao.org/docrep/T0848 S/t0848s00.htmContents. Fecha de consulta 1 de abril 2017.

INEGI (2010a) Red Hidrográfica. http://www.inegi.org.mx/geo/contenidos/recnat/hidrologia/Descarga.aspx. Fecha de consulta 1 de abril de 2017.

INEGI (2010b) Ortofotos 2000 y 2007. http://mapserver.inegi.org.mx/map/visortoDx/visor.html?c=1379\&s $=$ geo. Fecha de consulta 1 de abril de 2017. 
Li W, Wang Z, De Vriend HJ, Van Maren DS (2014) Long-Term Effects of Water Diversions on the Longitudinal Flow and Bed Profiles. Journal of Hydraulic Engineering 140 Doi: 10.1061/(ASCE)HY.19437900.0000856 .

Minea G, Zaharia L (2011) Geomorphological impact of floods in the Basca Catchment (Romania). Revista De Geomorfologie 13: 67-74.

Naoura J, Benaabidate L (2011) Monitoring of heavy metals in the sediments of the Inaouene River, Morocco. Journal of The Black Sea/Mediterranean Environment 17: 193-202.

Rosier B, Boillat JL, Schleiss AJ (2011) Influence of lateral water withdrawal on bed form geometry in a channel. Journal of Hydraulic Engineering 137: 1668-1675.

Wohl E (2015) Legacy effects on sediments in river corridors. Earth-Science Reviews 147: 30-53. 
